\section{Aportación española a la farmacia cubana decimonónica}

\section{Spain's contribution to pharmacy in $19^{\text {th }}$ century Cuba}

José Manuel González de la Peña Puerta

Doctorando en el Departamento de Farmacia y Tecnología Farmacéutica/Facultad de Farmacia/Universidad de Sevilla $\mathrm{C} /$ Paraíso, $6,5^{\circ} \mathrm{C}$

11405 Jerez de la Frontera (Cádiz) - España jgonzalezdelapena@redfarma.org

\section{Antonio Ramos Carrillo}

Profesor del Departamento de Farmacia y Tecnología Farmacéutica/ Facultad de Farmacia/Universidad de Sevilla C/ Porvenir 32-34, bloque 1, bajo C 41013 Sevilla - España

antonioramos@us.es

\section{Esteban Moreno Toral}

Profesor del Departamento de Farmacia y Tecnología Farmacéutica/ Facultad de Farmacia/Universidad de Sevilla

C/ Profesor García González, 2

41012 Sevilla - España

toral@us.es

Recibido para publicación en Noviembre 2006.

Aprobado para publicación en Abril 2008.
PEÑA PUERTA, José Manuel González de la; RAMOS CARRILLO, Antonio; MORENO TORAL, Esteban. Aportación española a la farmacia cubana decimonónica. História, Ciencias, Saúde - Manguinhos, Rio de Janeiro, v.15, n.2, p.487-496, abr.-jun. 2008.

\section{Resumen}

La llegada de los españoles a Cuba supuso una organización que fue un reflejo de la establecida en la metrópoli. En materia farmacéutica, se intentó regularizar el ejercicio de la misma y formar profesionales. Con este objetivo, entre otros, se constituyó en 1711 el Real Tribunal del Protomedicato de La Habana, sustituido en 1833 por las Reales Juntas Superiores Gubernativas de Medicina y Cirugía y de Farmacia hasta 1842 , cuando quedaron en manos de la Universidad de La Habana sus competencias en lo relativo a la enseñanza. Durante este tiempo, la Facultad de Farmacia en Cuba permaneció unida a la de medicina, hasta 1863. No podemos dejar sin mencionar la Real Academia de Ciencias Médicas, Físicas y Naturales de La Habana y las publicaciones científicas sobre farmacia de mediados de siglo.

Palabras clave: farmacia; Cuba; enseñanzas; universidad; siglo XIX.

\section{Abstract}

With the arrival of the Spanish in Cuba, society was organized along the lines of what already existed in the metropolis. In the area of pharmacy, this meant standardizing the practice of pharmacy and training professionals. It was with this and other purposes in mind that the Real Tribunal del Protomedicato de La Haban was established in 1711. In 1833 it was replaced by the Reales Juntas Superiores Gubernativas de Medicina y Cirugía y de Farmacia until 1842, when educational responsibilities were transferred to the Universidad de La Habana. Throughout this period, the Facultad de Farmacia in Cuba kept its links with the Facultad de Medicina, until 1863. Another contribution are the Real Academia de Ciencias Médicas, Físicas y Naturales de La Habana and the scientific publications on pharmacy in the midle the century.

Keywords: pharmacy; education; university; $19^{\text {th }}$ century; Cuba. 
$\mathrm{E}$ s sabido que, en 1492, Cristóbal Colón con una modesta tripulación y con el ideal de llegar a las Indias por el Oeste, llegó a un territorio desconocido que para él eran las Indias, pero que resultó ser un nuevo mundo para los europeos. Fue en este viaje cuando descubrió la isla de Cuba, aunque realizaría otros tres más con la intención de explorar e ir conquistando esas tierras. Pero, pasaron 17 años hasta que se decidió conquistar la isla debido a las riquezas en forma de metales preciosos que encontraron en islas vecinas y que no eran tan abundantes en Cuba.

Debido a que en el año 2006 se celebró el quinto centenario de la muerte de este brillante navegante, queremos aportar nuestra contribución acerca de uno de los aspectos sanitarios en la isla, la farmacia, centrándonos en los últimos cien años de dominación española.

El siglo XIX, tanto en Cuba como en España, fue un siglo de cambios. En España la Guerra de la Independencia, la alternancia de períodos constitucionales y de tipo absolutista, las diferentes revueltas de las corrientes liberales y demás cambios políticos, sumado al descontento existente en las colonias españolas en América, hicieron que muchas de éstas tomaran la iniciativa de independizarse y lo consiguieran. En Cuba, aunque también pervivía un espíritu independentista en la primera mitad de siglo no fue tan manifiesto, pues fue época de desarrollo económico, comercial y cultural, que ya venía floreciendo desde finales de la centuria anterior. Aparecieron grandes hacendados azucareros y cafetaleros, pero a la vez una gran diferencia de clases sociales, los ricos terratenientes, los campesinos y esclavos, lo que ocasionó cada vez más y más hostilidades entre ambos grupos. A todo esto hay que añadir las medidas represoras del gobierno colonial en pos de evitar estas discordias, las cuales fomentaron aún más odio a la metrópoli entre las clases más desfavorecidas agravando la situación. Diversas corrientes reformistas que fracasaron y un comienzo de lucha independentista desde distintos puntos de la isla, hicieron que el último tercio de siglo viniera marcado por un gran movimiento revolucionario y sucesivas guerras que se hacen patentes a partir de 1868 hasta alcanzar, con intervención también de Estados Unidos, la independencia en 1898 (Le Riverend, 1992, p.33-75).

En cuanto a lo que respecta a la farmacia, el siglo XIX fue un siglo de progreso, ya que sobre todo en su segunda mitad hubo avances de mucha repercusión. Un ejemplo lo puede constituir la microbiología, después ya de la llegada de la vacuna de la viruela, algo que sería de pronta aplicación en Cuba (concretamente en 1804 por el doctor Tomás Romay) debido a las epidemias sufridas en su mayoría por esclavos. También tuvo incidencia el despegue de la industria que hizo que la farmacia fuera dejando a un lado la elaboración artesanal del medicamento dando paso al medicamento ya preparado.

En Cuba, la farmacia era muy precaria, existía todavía una presencia de curanderos y charlatanes pero, gracias a la creación de la universidad (1728), y más tarde el establecimiento de la Real Junta Superior Gubernativa de la Facultad de Farmacia, la profesión adquirió más rigor científico y se dotó de farmacéuticos más preparados con un consiguiente crecimiento en el número de boticas en todo el territorio, lo cual repercutió en la salud de la población.

El primer boticario del que se tiene constancia fue el licenciado Gamarra, autorizado "para ejercer las tres ramas, medicina, cirugía y botica" el 26 de febrero de 1569 (López Sánchez, 1970a, p.10). Pero no es hasta el siglo. XIX cuando se observa un verdadero 
despegue de la farmacia. Según el artículo de Sonia Menéndez y Anicia Rodríguez (2001) sobre las farmacias habaneras en el diecinueve y las anotaciones de Manuel García Hernández y Susana Martínez-Fortún y Foyo (1967) en sus "Apuntes históricos relativos a la farmacia en Cuba", hasta 1834 las farmacias eran muy primitivas y pocas en número, pero a partir de entonces se van haciendo más lujosas, existiendo en 1885 unas sesenta y ocho farmacias.

\section{La regulación de la sanidad: El Real Tribunal del Protomedicato (1711-1833)}

El Real Tribunal del Protomedicato de España data del año 1477 y su creación se debió a los Reyes Católicos con el fin de ser un órgano que regulara las actividades sanitarias, entre ellas el ejercicio farmacéutico. Era una institución que se ocupaba, en este ámbito, de todas las actividades farmacéuticas, los exámenes y la concesión de licencias para poder ejercer de forma legal.

Este organismo se estableció en todos los dominios españoles de las Indias. Sin embargo, en Cuba pasarían muchos años hasta su establecimiento en 1634, gracias al médico español Francisco Muñoz de Rojas, graduado en Sevilla y residente en Cuba, el cual fue nombrado protomédico de la isla. Hasta entonces, todos los aspirantes a cirujanos, barberos, boticarios y parteras debían hacer su examen en Nueva España -actual Méjico - de cuyo protomedicato dependía la isla (Santovenia, 1952, p.15).

Así se hace constar en las Actas Capitulares del Ayuntamiento de La Habana que aparecen recogidas en la obra de José López Sánchez:

Lcdo. D. Francisco Muñoz de Rojas, he tenido y por la presente tengo por bien que ahora y de aquí en adelante cuanto mi voluntad fuese, seáis mi protomédico ecsaminador de todos los doctores, cirujanos, barberos, boticarios y parteras de la dicha ciudad de la Habana y Ysla de Cuba, y que como tal podáis usar y uséis el dicho cargo en los casos y cosas a él anejas y concernientes según y de la manera que lo usan y pueden y deben usar los protomédicos y ecsaminadores destos reinos y de la ciudad de Megico, Los Reyes, Santa Fe, y Cartagena, ecsaminando y dando títulos y licencias a los que hallárades hábiles y suficientes para los dichos oficios ... (López Sánchez, 1970a, p.63).

El tribunal existió mientras el Ldo. Muñoz de Rojas vivió. Tras su muerte desapareció, quedando la isla sin regulación ninguna y a merced de individuos no cualificados, en muchas ocasiones curanderos o charlatanes, hasta la llegada de Francisco Teneza y Rubira que, sin ser médico - pues era doctor en derecho civil -, pero de reconocida valía por sus destacadas acciones asistenciales y médicas en travesías navales, pudo ostentar el cargo de protomédico de La Habana y fundar el Real Tribunal del Protomedicato de La Habana en 1711, similar a los ya existentes en otras colonias españolas, y que estaría presente en la isla hasta 1833 (Santovenia, 1952, p.15-22).

La importancia de esta institución radica en que nos encontramos en una época de grandes carencias en materia sanitaria, pues en Cuba todavía no se estudiaba medicina, ni cirugía y eran pocos los médicos venidos de fuera. Esto lo podemos extrapolar al resto de profesionales sanitarios, abundando, como hemos expuesto, la presencia de prácticos y curanderos. 
Este tribunal se encargaba de examinar a boticarios, especieros y herbolarios, controlaba la venta de medicinas y a los médicos que invadían el campo de los farmacéuticos, algo que era bastante habitual en esta época.

De las Actas Capitulares del Ayuntamiento de La Habana (donde se recogen numerosas solicitudes de licencia para ejercer el arte farmacéutico por parte de maestros farmacéuticos con título expedido por el protomedicato, ya sea de La Habana o de Méjico) reunidas en la obra de López Sánchez, se desprende que en lo referente a los requisitos que debía cumplir un aspirante a boticario y a su examen, para ser examinado por el Real Tribunal del Protomedicato, era necesario haber estado cuatro años de práctica con un maestro boticario, además de presentar una serie de documentos como su fe de bautismo, limpieza de sangre, buena vida y costumbres, y tener cumplidos veinticinco años.

Cumplidos los requisitos, el candidato podía optar a examinarse. El aspirante era examinado de latinidad y teórica de dicho arte, donde se valoraba su conocimiento de las drogas; empleo y elección de yerbas; preparación y duración de las medicinas; modo de mezclar los compuestos y demás cuestiones concernientes al mencionado arte. En dicho examen se le daba un libro de farmacia, que normalmente era el libro de los Cánones de Mesué, y se le hacía leer y explicar un párrafo escogido por el tribunal. Posteriormente se le hacían diversas preguntas acerca de lo leído y de cualquier aspecto farmacéutico. También había una parte práctica, realizada en una botica, donde debía reconocer distintas medicinas y graduar distintos pesos, pesas y medidas, tras lo cual se tenía que enfrentar a un turno de preguntas.

Si estas pruebas eran superadas, se le permitía poner botica pública en cualquiera de los dominios de S.M. y se le hacía juramento de defender la Purísima Concepción de la Virgen María y de usar adecuadamente su facultad, además de pagar 'media annata' en concepto de derechos (López Sánchez, 1970b, p.56-62, 76-81, 95, 97, 110, 194, 205).

\section{La Real Junta Superior Gubernativa de Farmacia}

La Real Junta Superior Gubernativa de la Facultad de Farmacia apareció en España en el año de 1800 tras la desaparición del Real Tribunal del Protomedicato, el cual debido a los complejos cambios políticos que acontecieron en la España decimonónica volvió a restablecerse, aunque ya con una vida efímera. Al igual que el caso español, ésta también se instauró en Cuba en 1833, fruto de la existencia de un protomedicato cada vez más en decadencia y cuya ineficacia se pondría de manifiesto tras la aparición en la isla de una epidemia de cólera morbo-asiático. Esta Junta, según su Real Cédula de erección, era independiente de la Junta de Medicina y Cirugía y a su vez de las establecidas en España. Las atribuciones de la Junta eran gubernativo-económicas y entre sus funciones destacamos la de expedir los títulos de licenciado y doctor, nombrar visitadores e inspectores, establecer los precios de las medicinas, asimismo misión supervisora, administrativa, del control del intrusismo y, en general, todo lo tocante a la profesión. De ella se dice en uno de los artículos de su reglamento, que es "cabeza y gefe de la facultad de farmacia" (Reglamento..., 1834).

De esta forma, la farmacia quedaba controlada por farmacéuticos y no por médicos, como sucediera durante la existencia del protomedicato. 
En cuanto a la formación, la Junta estableció un plan de estudios de farmacia. Así se establecieron tres cátedras: farmacia teórica, química y botánica. Los estudios duraban un total de cinco años y quedaban divididos en tres años de teoría y dos de práctica. La parte teórica se estructuraba de la siguiente forma: los estudiantes comenzaban por la botánica, dividida en dos cursos, de marzo a junio el primero y de septiembre a diciembre el segundo. Las clases se impartían en el Jardín Botánico, aunque más tarde pasaron a impartirse en la universidad (esto para el primer año). Tras obtener la certificación de haber cursado botánica, en el segundo año se matriculaban en química, también estructurada en dos cursos, de septiembre a febrero el primero y de marzo a junio el segundo. Al igual que en el caso anterior, habiendo obtenido la certificación correspondiente de haber estudiado química, el alumno se matriculaba en farmacia teórica, constituyendo éste el último año de los estudios teóricos, y teniendo los mismos cursos que para la química.

Una vez concluidos los cursos, se graduaban de bachilleres en farmacia mediante un examen que hacía la Junta. Con este grado de bachiller, podían matricularse de práctica y, transcurridos los dos años de práctica y tras obtener su certificado, podían presentarse a examen ante la Junta, para así obtener el grado de licenciado, lo cual le confería la facultad de abrir oficina de farmacia.

También se contemplaba la opción de obtener el grado de doctor pero, para optar a ello, tenían que haber pasado como mínimo dos años después de haber recibido el de licenciado. Obviamente, cualquier alumno que deseare graduarse tenía que pagar unos derechos. Asî por ejemplo, para el grado de bachiller, las tasas eran de 34 pesos, 102 por el de licenciado y el doble por el de doctor. A esto hay que sumar una propina de tres pesos para cada examinador de bachiller y cuatro para los de licenciado y doctor (Reglamento..., 1834).

\section{La Universidad de La Habana y los estudios de farmacia}

Para hacer una evolución cronológica sobre los estudios en la universidad y particularmente los de farmacia, haremos una división en cuatro períodos: el primero desde su fundación hasta su secularización; el segundo desde su secularización hasta 1863; un tercero desde 1863 a 1880; y un cuarto desde 1880 hasta el fin del colonialismo español.

La Universidad de La Habana se fundó en 1728 por los religiosos de la Orden de Predicadores, también conocidos como dominicos, bajo el nombre de Real y Pontificia Universidad de San Jerónimo de La Habana y estaba ubicada en el Convento de San Juan de Letrán. Durante este primer período existían cinco facultades: Teología, Cánones (derecho canónico), Leyes (derecho civil), Medicina y Artes (filosofía). Había veinte cátedras, cuyos catedráticos se renovaban cada seis años, 16 de ellas formaban parte de esas cinco facultades y las otras cuatro restantes eran independientes, entre ellas Matemática y Gramática. En todas las facultades se otorgaban los grados de bachiller (grado menor), licenciado y doctor (grados mayores), excepto en la de Filosofía en la que se confería el grado de maestro en lugar del de doctor. Para la obtención de dichos grados, era necesaria la realización de unos ejercicios que solían ser orales. Es curioso destacar que los ejercicios para la obtención de grados mayores eran anunciados mediante repique de campana y el acto conducente al 
rango de doctor era de gran pompa y boato. También se había de acreditar mediante documentos la legitimidad, limpieza de sangre y la buena vida y costumbres (Armas, TorresCuevas, Cairo Ballester, 1984, p.49, 52, 53).

Tras varios intentos de reforma fallidos, en 1842 se seculariza la universidad recibiendo el nombre de Real y Literaria Universidad de La Habana, estableciéndose un nuevo plan de estudios y reglamento. Con este plan, la enseñanza que se daría en la universidad correspondería a la secundaria elemental y superior, y la de facultades, que serían Jurisprudencia, Medicina, Cirugía y Farmacia.

Vemos como ya en este segundo plan de estudios aparece como tal la Facultad de Farmacia. De esta forma, la formación farmacéutica se va cimentando en continuidad con la labor de reglamentación de la profesión y regularización de su estudio llevada a cabo por la citada Real Junta Superior Gubernativa de la Facultad de Farmacia.

En esta Facultad se estudiaban dos años de teoría: en el primer año se cursaban las asignaturas de elementos de farmacia teórica y química aplicada a la medicina y a la farmacia, teniendo que asistir además a la clase de química de la Universidad; en el segundo, las de farmacia experimental y práctica y botánica. Habiendo cursado estos dos años de teoría, era necesario hacer dos años de práctica para licenciarse y, durante los mismos, en el primer año de práctica se exigía repetir la asistencia a la clase de farmacia experimental y en el segundo a la de materia médica y arte de recetar de la Facultad de Medicina (Memoria..., 1870). Se puede observar como los estudios de farmacia se encontraban asociados a los de medicina, pues algunas de sus asignaturas se impartían en dicha facultad.

$\mathrm{Al}$ igual que en el anterior período se conferían grados de bachiller, licenciado y doctor. El grado de bachiller en artes o ciencias era otorgado por la Facultad de Filosofía y equivalía a haber cursado los estudios de enseñanza superior. Esto era requisito para poder matricularse en cualquier facultad de las llamadas mayores, entre ellas la de farmacia. Para la obtención de dichos grados, los aspirantes debían de someterse a diversos exámenes o pruebas más o menos complicadas según el grado, siendo los de licenciado los más difíciles. Asimismo, el aspirante debía de pagar unos derechos a examen que eran distintos según el grado (Armas, TorresCuevas, Cairo Ballester, 1984, p.140-142).

El tercer período que tratamos es el coincidente con el plan de estudios de 1863. Este plan libera de la Universidad todos los estudios de segunda enseñanza, creándose los Institutos de Segunda Enseñanza, en los que se expedía el título de bachiller en artes, requisito que seguía siendo indispensable para comenzar los estudios en la facultad.

En 1863 la Facultad de Farmacia se desligó de la de Medicina y Cirugía de la que dependía desde 1842. Podemos decir que, en este punto, es cuando los estudios de farmacia se asientan firmemente, siendo ya totalmente independientes y habiendo tomado ya toda su forma académica.

El nuevo plan estableció las siguientes asignaturas para la Facultad de Farmacia: materia farmacéutica correspondiente a los reinos animal y mineral; farmacia químico-inorgánica; farmacia químico-orgánica; ejercicios prácticos de reconocimiento y clasificación de objetos de materia farmacéutica; práctica de operaciones farmacéuticas; análisis químico aplicado a las ciencias médicas; e historia crítico-literaria de la farmacia. Además seguían dándose los grados de bachiller, licenciado y doctor (Memoria..., 1870). 
Este es un período, al igual que lo fue el de 1880, que estuvo marcado por los comienzos de la independencia cubana. Coincidiendo con la insurrección en Yara, en 1868 (primer choque con las tropas españolas), que fue el comienzo de una guerra que duró diez años, el gobernador de la isla, Blas Villate, promulgó una reforma en el plan de estudios de 1863 que consistió en suprimir los estudios de doctorado para varias facultades, entre ellas la de Farmacia. Pero el objetivo último encubría fines políticos más que universitarios, dada la situación que se vivía en la isla. Fue una medida de represión, pues se acusó a la Universidad y al profesorado de "convertir la cátedra de la ciencia en una tribuna revolucionaria" (Marchante Castellanos, Merchán González, sept.-dic. 2007). No obstante esas acusaciones tenían poco sentido debido a que la Universidad dependía del gobierno de la isla y del de la península, por lo que simplemente fueron medidas políticas. De hecho, cuando finalizó la guerra se restableció el doctorado, según el real decreto de 22 de febrero de 1879, aunque durante este período la vida universitaria se desarrolló bajo la influencia del estado de guerra existente en Cuba, lo que se reflejó también en un menor número de estudiantes (Marchante Castellanos, Merchán González, sept.-dic. 2007).

El cuarto y último período que tratamos corresponde, como hemos comentado, al plan de estudios de 1880, hasta la pérdida de la colonia española. El plan es consecuencia del real decreto dado en Madrid en 18 de junio de 1880, por el cual se implantaba en la isla el mismo régimen de estudios que existía en España, equiparándose los mismos. Durante estos años de gran agitación política y revolucionaria se sucedieron numerosos decretos, leyes y órdenes que van a ir modificándolo. Entre ellos podemos hacer referencia al decreto de 28 de julio de 1887, por el que se reforma la enseñanza de farmacia en la Universidad (Armas, Torres-Cuevas, Cairo Ballester, 1984, p.188-193).

Los estudios de la Facultad de Farmacia por este decreto quedaron estructurados en tres períodos: período preparatorio, período de licenciatura y período de doctorado. El primero de ellos se cursaría en la Facultad de Ciencias, constando de asignaturas como ampliación de la física, química general, mineralogía y botánica, y zoología. El segundo período, el de la licenciatura, contaría con botánica, mineralogía, zoología, materia farmacéutica vegetal, químicas orgánica e inorgánica, todas con sus prácticas correspondientes, análisis con sus prácticas, instrumentación, legislación y farmacia práctica. Y el de doctorado, con química biológica con su análisis e historia crítica de la farmacia y bibliografía farmacéutica (Gaceta..., 31 jul. 1887). La tesis titulada El método general adoptado hoy para la investigación de los alcaloides, presentada el cinco de junio de 1880, correspondió al primer grado de doctor en farmacia concedido por la Universidad después del decreto de 1871 (Marchante Castellanos, Merchán González, sept.-dic. 2007).

Se seguía requiriendo el bachiller en artes para comenzar el período preparatorio y además era necesario presentar un certificado de haber aprobado oficialmente un curso de lengua francesa. En el decreto también se establecía el orden a seguir en el estudio de todas las asignaturas mencionadas, ya que, por ejemplo, no era posible examinarse de materia farmacéutica vegetal antes que de botánica descriptiva.

Los exámenes de licenciatura eran orales ante un tribunal, donde al aspirante se le realizaban diversas preguntas, determinar ciertas plantas, analizar la pureza de un medicamento propuesto por el tribunal y la preparación de alguno. Para la obtención del 
José Manuel González de la Peña Puerta, Antonio Ramos Carrillo, Esteban Moreno Toral

grado de doctor se realizaba una tesis que, al igual que en la actualidad, se defendía ante un tribunal (Gaceta..., 31 jul. 1887).

Tras la independencia de Cuba desapareció la Real y Literaria Universidad de La Habana, para dar paso a la nueva Universidad de La Habana, comenzando así otro capítulo de su historia.

\section{La Real Academia de Ciencias Médicas, Físicas y Naturales de La Habana}

La Real Academia de Ciencias Médicas, Físicas y Naturales de La Habana se fundó en 1861 gracias al doctor Nicolás Gutiérrez Hernández quien, tras muchas trabas, consiguió el establecimiento de esta institución científica que vino a ser un gran apoyo al movimiento científico cubano del siglo XIX. En ella se discutían temas relacionados con la medicina, farmacia, química y la historia natural. Contribuyó al desarrollo científico, propiciando el debate de muchos temas sanitarios, instigando a científicos e intelectuales a la investigación, formando en los avances y descubrimientos del extranjero, lo cual evitó el atraso científico y fomentó el progreso. Algo verdaderamente importante si nos paramos a pensar que nos desenvolvemos en una época marcada por luchas independentistas, reformismo y una cierta hostilidad hacia la metrópoli (Gelpi Leyva, 2005).

Entre los temas tratados es de destacar la contribución del doctor Carlos J. Finlay, con sus teorías sobre la propagación de enfermedades debido a vectores biológicos. Esto supuso un gran adelanto sobre todo en el conocimiento de la transmisión de la fiebre amarilla, enfermedad muy común en esas zonas tropicales. Otros temas estarían relacionados con la higiene, la salud, e incluso encontramos algunos eminentemente farmacéuticos como el del caso de los llamados Polvos de Hita, un medicamento que era vendido como milagroso, pero que resultaba ser un fraude (Pruna, Goodgall, 2002, p.197-203).

También no debemos olvidar la publicación desde el año 1864 de Anales de la Academia de Ciencias Médicas, Físicas y Naturales de La Habana, que contribuyó a la difusión del conocimiento y donde aparecieron artículos de científicos extranjeros, originales y traducidos, y trabajos e investigaciones sobre diversas materias.

\section{Publicaciones sobre farmacia en Cuba}

Existen algunas publicaciones sobre farmacia y medicina en la segunda mitad del siglo XIX que no debemos dejar en el olvido porque constituyen un testimonio inestimable de todos los hallazgos, avances, investigaciones y opiniones sobre estas materias en dicho siglo. Hay antecedentes y obras de grandes personalidades, sobre todo de la medicina cubana, como Carlos J. Finlay o Tomas Romay, quienes ya en el siglo XVIII habían escrito sobre sus estudios acerca del "vómito negro" o fiebre amarilla, y son auténticos precursores de una bibliografía científico-médica que se irá poco a poco consolidando con todas las revistas que aparecieron en esta segunda mitad del siglo XIX (López Espinosa, ene.-jun. 2000).

Destacaremos algunas que, por su interés, consideramos más importantes. Una de ellas es La Emulación, una revista creada hacia 1863 y que, hasta 1867, se publicaron artículos de científicos de la propia isla y del extranjero, biografías de químicos, farmacéuticos o 
naturalistas. También hallamos temas sobre enfermedades como el cólera o la fiebre amarilla, o bien otros como terapéutica, higiene, etc. Repertorio de Farmacia es otra revista que comenzó a publicarse a partir de 1880 hasta 1885. En ella escribieron numerosos farmacéuticos y médicos sobre distintos temas de interés. Curioso es el Boletín de la Farmacia El Amparo, aparecido en 1884 y publicado hasta 1910, donde el propietario de dicha farmacia, Anselmo Castells Berri, anunciaba distintos productos farmacéuticos fabricados en su farmacia, además de distintos artículos sobre terapéutica de revistas extranjeras (López Espinosa, 2003).

Otras fueron Repertorio Médico Farmacéutico y de Ciencias Auxiliares, que apareció en 1890, y el Boletín del Colegio de Farmacéuticos de La Habana de 1895, que contemplaba la situación de la farmacia en Cuba y en España. A mayor abundamiento, citaremos algunas más generales o médicas como El Estímulo, Anales de la Academia de Ciencias Médicas, Físicas y Naturales de La Habana y la Gaceta de Ciencias Médicas, entre otras.

\section{Conclusiones}

Con estas líneas hemos querido hacer un recorrido de lo que fue la farmacia cubana durante el siglo XIX, centrándonos principalmente, como se ha podido observar, en el aprendizaje, las enseñanzas o el estudio de la misma en la isla. Hemos intentado aunar en el presente artículo y de forma muy resumida toda la información, bastante dispersa por otra parte, que hemos ido encontrando en los distintos documentos, libros, publicaciones y revistas que hemos consultado, entresacando lo concerniente a la farmacia y, concretamente, a la enseñanza de la misma.

Hemos estructurado este trabajo cronológicamente, comenzando con el Real Tribunal del Protomedicato, que abarca hasta el año 1833; siguiendo con la Real Junta Superior Gubernativa de la Facultad de Farmacia, donde ya toma forma la farmacia, independiente de la medicina; y la Universidad, donde aparecerían los estudios de farmacia en 1842, consolidándose como facultad independiente en 1863. No hemos querido dejar atrás la Real Academia de Ciencias Médicas, Físicas y Naturales de La Habana, por ser un centro que se crea a mediados del siglo XIX y que contribuye a la mejora de conocimientos, no solo en la farmacia sino en otras disciplinas. Y, por último, mencionar algunas publicaciones relacionadas con el tema por considerar que fueron también una fuente de enseñanza entre los farmacéuticos cubanos.

De todo esto se puede concluir que la presencia española - de todos sabido hasta 1898 - dotó a la isla de los organismos e instituciones que van apareciendo en España en este siglo, al menos, en lo que a la farmacia corresponde. Aunque irán llegando con una cierta demora, aun así le permitirá en esta centuria estar más avanzada que en sus naciones vecinas en cuanto a los estudios universitarios propiamente dichos, la ordenación de la profesión y a otros aspectos.

Se advierte que durante este siglo, y sobre todo en su segunda mitad, hay un gran auge de la profesión farmacéutica, porque gracias al establecimiento de la Universidad comienzan a formarse farmacéuticos en la propia isla, aumentando su número y resolviéndose así los problemas de curanderismo, intrusismo profesional y escasez de farmacias, entre otros, ya que hasta entonces los farmacéuticos existentes habían venido de España o tenían que 
formarse fuera. Éstos ejercerán una labor sanitaria muy importante en un período marcado por conflictos. Todo va a ir paralelo a la evolución socio-económica y científica que se va ir experimentando en Cuba y que culminará con un sentimiento de nacionalidad, una antipatía hacia lo español y finalmente, su independencia.

\section{REFERENCIAS}

ARMAS, Ramón de; TORRES-CUEVAS, Eduardo; CAIRO BALLESTER, Ana.

Historia de la Universidad de La Habana (17281929). v. 1. La Habana: Editorial de Ciencias Sociales. 1984.

\section{GACETA...}

Gaceta de La Habana. Habana (Archivo General de Indias/Archivo Histórico Nacional de Madrid/Ultramar, 273, exp. 46). 31 jul. 1887.

GARCÍA HERNÁNDEZ, Manuel; MARTÍNEZFORTÚN Y FOYO, Susana.

Apuntes históricos relativos a la farmacia de Cuba. Cuadernos de Historia de la Salud Pública, La Habana, n.33, p.19. 1967.

GELPI LEYVA, Ana María.

La Real Academia de Ciencias Médicas, Físicas y Naturales y su contribución al desarrollo de la ciencia colonial. Monografía. Facultad de Ciencias Médicas Dr. Salvador Allende, La Habana.o 2005. Disponible en: http:// www.ilustrados.com/publicaciones/ EEEEEAupEZyrjxWvyL.php\#superior

LE RIVEREND, Julio.

Breve historia de Cuba. La Habana: Editorial de Ciencias Sociales. 1992.

LÓPEZ ESPINOSA, José Antonio.

Contribución a la historia de la bibliografía farmacéutica cubana. Monografía - Centro Nacional de Información de Ciencias Médicas, Universidad Virtual de Salud, Cuba. Disponible en: http://educacionmedica.pri.sld.cu/datas/ historia/farmaceutica.html. 2003.

LÓPEZ ESPINOSA, José Antonio.

Revistas médicas surgidas en Cuba durante la sexta década del siglo XIX. Revista Cubana de Salud Pública, La Habana, v.25, n.1, p.68-77. Disponible en: http://www.bvs.sld.cu/revistas/ spu/vol26_1_00/spu10100.htm. ene.-jun. 2000.

LÓPEZ SÁNCHEZ, José.

La medicina en La Habana, 1550-1730: cronología de los hechos médicos consignados en las Actas Capitulares del Ayuntamiento de
La Habana, 1. parte. Cuadernos de Historia de la Salud Pública, La Habana, n.47. 1970a.

LÓPEZ SÁNCHEZ, José.

La medicina en La Habana, 1731-1799: cronología de los hechos médicos consignados en las Actas Capitulares del Ayuntamiento de La Habana, 2. parte. Cuadernos de Historia de la Salud Pública, La Habana, n.48. 1970b.

MARCHANTE CASTELLANOS, Pilar; MERCHÁN GONZÁLEZ, Francisco. Enseñanza de la farmacia en la Real Universidad de La Habana según Plan de Estudios de 1863: etapa 1871-1880. Revista Cubana de Farmacia, La Habana, v.41, n.3, p.120. Disponible en: http://bvs.sld.cu/revistas/far/ vol41_3_07/far13307.htm. sept.-dic. 2007.

\section{MEMÓRIA....}

Memoria acerca del estado de la enseñanza en la Universidad de La Habana en el curso de 1868 a 1869. Habana. (Archivo General de Indias/ Archivo Histórico Nacional de Madrid/ Ultramar, 272, exp.3). 1870.

MENÉNDEZ, Sonia; RODRÍGUEZ, Anicia. Farmacias habaneras. Opus Habana, La Habana, v.5, n. 2, p.1-5. Disponible en: http:// www.habananuestra.cu/index.php?option= com_content\&task=view\&id=240\&Itemid=26\& limit $=1 \&$ limitstart $=1.2001$.

PRUNA GOODGALL, Pedro M. La Real Academia de Ciencias de La Habana, 1861-1898. Madrid: CSIC. 2002.

\section{REGLAMENTO...}

Reglamento para la Dirección y Gobierno de la Real Junta Superior Gubernativa de la Facultad de Farmacia. Habana. (Archivo General de Indias/ Archivo Histórico Nacional de Madrid/ Ultramar, 14, exp.16). 1834.

SANTOVENIA, Emeterio S. El protomedicato de La Habana. Cuadernos de Historia Sanitaria, La Habana, n.1, p.15-22. 1952.

\section{$\rightarrow \rightarrow+<<$}

